\title{
STATUS AND PROSPECTS FOR LORENTZ AND CPT VIOLATION TESTS AT KLOE AND KLOE-2
}

\author{
ANTONIO DE SANTIS \\ Dipartimento di Fisica, Università di Roma 'La Sapienza' \\ and I.N.F.N. Sezione di Roma \\ P.le A. Moro, 2 \\ I-00185 Rome, Italy \\ E-mail: antonio.desantis@roma1.infn.it
}

on behalf of the KLOE and KLOE-2 collaborations

\begin{abstract}
The neutral kaon system offers a unique possibility to perform fundamental tests of CPT invariance. In this contribution the KLOE prospects for the measurements of CPT violation in the context of the Standard-Model Extension are presented together with a full description of the analysis method needed and with the perspective given by the KLOE- 2 data-taking campaign.
\end{abstract}

\section{The KLOE experiment}

The KLOE experiment operates at DA $\Phi$ NE, the Frascati $\phi$-factory. $\mathrm{DA} \Phi \mathrm{NE}$ is an $e^{+} e^{-}$collider running at a center of mass energy of $\sim 1020$ $\mathrm{MeV}$, the mass of the $\phi$ meson. Positron and electron beams collide at an angle of $\pi-25 \mathrm{mrad}$, producing $\phi$ mesons with small boost in the orbit plane $\left(p_{x}(\phi) \sim-15 \mathrm{MeV}\right)$.

The KLOE detector consists of a large cylindrical drift chamber (DC) surrounded by a lead-scintillating fiber electromagnetic calorimeter (EMC). A superconducting coil around the EMC provides a $0.52 \mathrm{~T}$ axial field. The $\mathrm{DC}^{1}$ is $4 \mathrm{~m}$ in diameter and $3.3 \mathrm{~m}$ long and has 12,582 all-stereo tungsten sense wires. The chamber shell is made of carbon fiber-epoxy composite and the gas used is a $90 \%$ helium, $10 \%$ isobutane mixture. These features maximize transparency to photons and reduce $K_{L} \rightarrow K_{S}$ regeneration and multiple scattering. The position resolutions are $\sigma_{r, \phi} \sim 150 \mu \mathrm{m}$ and $\sigma_{z} \sim 2$ $\mathrm{mm}$. The momentum resolution is $\sigma\left(p_{\perp}\right) / p_{\perp} \sim 0.4 \%$. The calorimeter ${ }^{2}$ is divided into a barrel and two endcaps, and covers $98 \%$ of the solid angle. The modules are read-out at both ends by photomultipliers, both in am- 
plitude and time for a total of 2440 cells per side arranged in five layers. Cells close in time and space are grouped into calorimeter clusters. The cluster energy $E$ is the sum of the cell energies. The cluster time $T$ and position $\vec{R}$ are energy-weighed averages. Energy and time resolutions are $\sigma_{E} / E=5.7 \% / \sqrt{E(\mathrm{GeV})}$ and $\sigma_{t}=57 \mathrm{ps} / \sqrt{E(\mathrm{GeV})} \oplus 100 \mathrm{ps}$, respectively.

Actually KLOE has already acquired $2.5 \mathrm{fb}^{-1}$ of data and a new extensive campaign of data taking is starting aiming at an integrated luminosity of $25 \mathrm{fb}^{-1}$.

The cross section for the process $e^{+} e^{-} \rightarrow \phi$ production is $3.3 \mu \mathrm{b}$ and the $\phi$ meson decays into $K_{0} \bar{K}_{0}$ with a branching fraction of $\sim 34 \%$. The initial state of the kaon pair is produced via strong interaction with quantum numbers $J^{P C}=1^{--}$:

$$
|i\rangle=\frac{\left|K_{0}\right\rangle\left|\bar{K}_{0}\right\rangle-\left|\bar{K}_{0}\right\rangle\left|K_{0}\right\rangle}{\sqrt{2}}=\mathcal{N}\left(\left|K_{S}\right\rangle\left|K_{L}\right\rangle-\left|K_{S}\right\rangle\left|K_{L}\right\rangle\right),
$$

where $\left|K_{S} / K_{L}\right\rangle=\left(1+\varepsilon_{S / L}\right)\left|K_{0}\right\rangle \pm\left(1-\varepsilon_{S / L}\right)\left|\bar{K}_{0}\right\rangle$ and $\varepsilon_{S / L}=\varepsilon \pm \delta$. The two kaons are in an antisymmetric correlated state and the time evolution of their state in two different final states $f_{1}$ and $f_{2}$ for the two kaons as a function of the difference of decay time $\left(\Delta t=t_{2}-t_{1}\right)$ can be expressed as the decay intensity as a function of $\Delta t$ :

$$
\begin{aligned}
I_{f_{1} f_{2}}(\Delta t) \propto & \left|\eta_{1}\right| e^{-\Gamma_{L} \Delta t}+\left|\eta_{2}\right| e^{-\Gamma_{S} \Delta t} \\
& -2 e^{-\frac{\left(\Gamma_{S}+\Gamma_{L}\right)}{2} \Delta t} \cos \left(\Delta m \Delta t+\phi_{2}-\phi_{1}\right),
\end{aligned}
$$

where

$$
\eta_{j}=\left\langle f_{j} \mid K_{L}\right\rangle /\left\langle f_{j} \mid K_{S}\right\rangle=\left|\eta_{j}\right| e^{i \phi_{j}}
$$

\section{CPT and Lorentz symmetry breaking}

A general theoretical possibility for CPT violation is based on spontaneous breaking of Lorentz symmetry, as developed by Kostelecký: ${ }^{3-5}$ the Standard-Model Extension (SME).

In SME for neutral kaons, CPT manifests to lowest order only in the CPT violation parameter $\delta$, and exhibits a dependence on the 4-momentum of the kaon:

$$
\delta \approx i \sin \phi_{S W} e^{i \phi_{S W}} \gamma_{K}\left(\Delta a_{0}-\vec{\beta}_{K} \cdot \Delta \vec{a}\right) / \Delta m,
$$

where $\gamma_{K}$ and $\vec{\beta}_{K}$ are the kaon boost factor and velocity in the observer frame, and $\Delta a_{\mu}$ are the CPT violation coefficients for the two valence quarks in the kaon. 
The time dependence arising from the rotation of the Earth can be explicitly displayed in Eq. (4) by choosing a three-dimensional basis $(\hat{X}, \hat{Y}, \hat{Z})$ in a nonrotating frame, with the $\hat{Z}$ axis along the Earth's rotation axis, and a basis $(\hat{x}, \hat{y}, \hat{z})$ for the laboratory frame. ${ }^{4}$ The CPT violating parameter $\delta$ may then be expressed as:

$$
\begin{aligned}
\delta\left(\vec{p}_{K}, t\right)=\frac{i \sin \phi_{S W} e^{i \phi_{S W}}}{\Delta m} \gamma_{K}[ & \Delta a_{0}+\beta_{K} \Delta a_{Z}(\cos \vartheta \cos \chi-\sin \vartheta \cos \phi \sin \chi) \\
& -\beta_{K} \Delta a_{X} \sin \vartheta \sin \phi \sin \Omega t \\
& +\beta_{K} \Delta a_{X}(\cos \vartheta \sin \chi+\sin \vartheta \cos \phi \sin \chi) \cos \Omega t \\
& +\beta_{K} \Delta a_{Y}(\cos \vartheta \sin \chi+\sin \vartheta \cos \phi \sin \chi) \sin \Omega t \\
& \left.-\beta_{K} \Delta a_{Y} \sin \vartheta \sin \phi \cos \Omega t\right],
\end{aligned}
$$

where $t$ is the sidereal time, $\Omega$ is the Earth's sidereal frequency and $\chi$ is the angle between the Earth rotation axis and the $\hat{z}$ direction in the laboratory frame.

Since inside the KLOE detector, kaons are produced in almost all direction, it is possible to use the experiment like a telescope to explore any direction in the space by using two different method to measure the $\Delta a_{\mu}$ parameters of Eq. (4):

- by studying the sidereal time variation of the semileptonic asymmetry of the $K_{L}$ and $K_{S}$. A preliminary measurement of the $\Delta a_{0}$ has been already performed with KLOE data using the difference between the semileptonic asymmetries of $K_{S}$ and $K_{L}$ : $\Delta a_{0}=(0.4 \pm 1.8) \times 10^{-17} \mathrm{GeV}{ }^{6}$

- by studying the quantum interferometry between the two kaons, Eq. (2). In this case, two different final state can be considered: $\left(\pi l^{+} \nu, \pi l^{-} \nu\right)$ and $\left(\pi^{+} \pi^{-}, \pi^{+} \pi^{-}\right)$.

In the following we will focus on interferometry with the channel $\phi \rightarrow K_{S} K_{L} \rightarrow \pi^{+} \pi^{-} \pi^{+} \pi^{-}$. Detailed discussion concerning other methods can be found elsewhere. ${ }^{6}$ Using this channel the two kaons decay both in the same final state, but have opposite momenta and experience different direction in the space. For instance, we can distinguish between the two kaons by their forward or backward emission. In this way it is possible to define as $K_{+}\left(K_{-}\right)$the one going in the forward (backward) direction with $\cos (\vartheta)>(<) 0$. The ratio of amplitudes in Eq. (3) is $\eta_{ \pm}=\varepsilon-\delta(\vec{p})$. Since the kaon momenta are opposite, a small asymmetry in the decay intensity 
$I_{ \pm}(\Delta t)$ will appear and the following ratio will be different from zero:

$$
A(\Delta t)=\frac{I_{ \pm}(\Delta t>0)-I_{ \pm}(\Delta t<0)}{I_{ \pm}(\Delta t>0)+I_{ \pm}(\Delta t<0)} .
$$

The above asymmetry for $\Delta t \gg \tau_{S}$ tends to zero, because $\varepsilon$ and $\delta$ are $90^{\circ}$ out of phase: ${ }^{7}$

$$
A\left(\Delta t \tau_{S}\right) \simeq-2 \Re\left(\frac{\delta}{\epsilon}\right) \sim 0
$$

while for $|\Delta t| \leq 5 \tau_{S}$

$$
A\left(|\Delta t| 5 \tau_{S}\right) \propto-2 \Im m\left(\frac{\delta}{\epsilon}\right)
$$

and therefore to $\Delta a_{X, Y, Z}$.

With analysis based on $1 \mathrm{fb}^{-1}$ we get a preliminary result on $\Delta a_{X, Y, Z}:^{8}$

$$
\begin{array}{r}
\Delta a_{X}=(-6.3 \pm 6.0) \times 10^{-18} \mathrm{GeV} \\
\Delta a_{Y}=(2.8 \pm 5.9) \times 10^{-18} \mathrm{GeV} \\
\Delta a_{Z}=(2.4 \pm 9.7) \times 10^{-18} \mathrm{GeV} .
\end{array}
$$

This analysis scheme using the simple forward backward asymmetry is not sensitive to $\Delta a_{0}$ and its effect is washed out in the asymmetry Eq. (5) and Eq. (6). As shown in Eq. (4) this parameter is coupled only with the $\gamma_{K}$ factor. Since at DA $\Phi N E$ the $\phi$ is produced with a small boost in the horizontal plane the kaons have different values for $\gamma_{K}$ as a function of the

azimuthal angle. The ratio of the decay intensities $I_{ \pm}(\Delta t)$ distribution for events in which the $K_{+}$propagates opposite or along the $\phi$ momentum will enhance the small asymmetry introduced by the $\Delta a_{0}$ component of the $\delta$ parameter. For values of $\Delta a_{0}$ of the order $\mathcal{O}\left(10^{-18}\right)$ we expect an effect on the $I_{ \pm}(\Delta t)$ up to $1 \%-2 \%$ in the region $|\Delta t|<5 \tau_{S}$ from which we expect to be able to put limits of the order $\mathcal{O}\left(10^{-17}\right)-\mathcal{O}\left(10^{-18}\right)$.

The $\Delta a_{\mu}$ parameters can be all simultaneously measured by performing a proper sidereal time dependent analysis of asymmetries of $I_{ \pm}(\Delta t)$, Eq. (2) and Eq. (5). An accuracy $\mathcal{O}\left(10^{-18}\right) \mathrm{GeV}$ could be reached with the analysis of the full KLOE data sample.

\section{Conclusions and future plans}

All four $\Delta a_{\mu}$ parameters of the SME can be independently measured at KLOE, completing results obtained by fixed beam experiments.

The continuation of the KLOE physics program with KLOE- $2^{9}$ at an improved DA $\Phi N E$ machine is currently starting. The data taking campaign 
will be organized in two different steps. During the first one we will double the statistics already taken by KLOE with a new beam interaction scheme ${ }^{10}$ and with the inclusion of two pairs of electron-positron taggers ${ }^{11}$ for the study of the gamma-gamma physics. The second phase aims to an integrated luminosity of $\sim 25 \mathrm{fb}^{-1}$ including several upgrades for the KLOE detector:

- a pair of crystal calorimeters $\left(\mathrm{CCALT}^{12}\right)$ near the interaction region to improve the angular acceptance for low- $\theta$ particles;

- a pair of tile calorimeters $\left(\mathrm{QCALT}^{13}\right)$ covering the quadrupoles along the beam pipe made of tungsten foil and singly read-out scintillator tiles to improve the angular coverage for particles coming from the active volume of the DC;

- a small and light tracker (IT ${ }^{14}$ ) made of four planes of cylindrical GEM to improve the resolution of the vertex reconstruction around the interaction point and to increase the low- $\theta$ charged particles acceptance.

One of the main physics issue of KLOE-2 is the search for CPT violation effects; limits on several parameters are expected to be improved by about one order of magnitude.

\section{References}

1. M. Adinolfi et al., KLOE Collaboration, Nucl. Inst. Meth. A 488 (2002) 51.

2. M. Adinolfi et al., KLOE Collaboration Nucl. Inst. Meth. A 482 (2002) 363.

3. V.A. Kostelecký, Phys. Rev. Lett. 80, 1818 (1998).

4. V.A. Kostelecký, Phys. Rev. D 61, 016002 (1999).

5. V.A. Kostelecký, Phys. Rev. D 64, 076001 (2001).

6. A. Di Domenico, in V.A. Kostelecký, ed., Fourth Meeting on CPT and Lorentz Symmetry, World Scientific, Singapore, 2008; A. Di Domenico, in A. Di Domenico ed., Handbook on neutral kaon interferometry at a $\Phi$-factory, Frascati Physics Series, Vol. 43.

7. C. D. Buchanan et al., Phys. Rev. D 45, 4088 (1992).

8. A. Di Domenico, J. Phys. Conf. Ser. 171, 012008 (2009).

9. G. Amelino-Camelia et al., arXiv:1003.3868.

10. C. Milardi et al., DAFNE Collaboration, arXiv:1006.1487.

11. D. Babusci et al., Nucl. Instrum. Meth. A 617, 81 (2010).

12. F. Happacher et al., Nucl. Phys. Proc. Suppl. 197, 215 (2009).

13. M. Cordelli et al., Nucl. Instrum. Meth. A 617, 105 (2010).

14. F. Archilli et al., KLOE-2 Collaboration, arXiv:1002.2572. 